\title{
Combined exercise and cognitive behavioral therapy improves outcomes in patients with heart failure
}

\author{
Rebecca A. Gary ${ }^{a, *}$, Sandra B. Dunbar ${ }^{a}$, Melinda K. Higgins ${ }^{a}$, \\ Dominique L. Musselman ${ }^{b}$, Andrew L. Smith ${ }^{\mathrm{c}}$ \\ ${ }^{\mathrm{a}}$ Nell Hodgson Woodruff School of Nursing, Emory University, Atlanta, GA 30322, USA \\ ${ }^{\mathrm{b}}$ Department of Psychiatry, School of Medicine, Emory University, Atlanta, GA 30322, USA \\ ${ }^{\mathrm{c}}$ Department of Cardiology, School of Medicine, Emory University, Atlanta, GA 30322, USA
}

Received 20 June 2009; received in revised form 15 January 2010; accepted 15 January 2010

\begin{abstract}
Objective: The purpose of this study is to compare the effectiveness of a combined 12-week home-based exercise (EX)/ cognitive behavioral therapy (CBT) program $(n=18)$ with CBT alone $(n=19), \mathrm{EX}$ alone $(n=20)$, and with usual care (UC, $n=17)$ in stable New York Heart Association Class II to III heart failure (HF) patients diagnosed with depression. Methods: Depressive symptom severity [Hamilton Rating Scale for Depression (HAM-D)], physical function [6-min walk test (6MWT)], and health-related quality of life (HRQOL) (Minnesota Living with Heart Failure Questionnaire) were evaluated at baseline (T1), after the 12-week intervention/control (T2), and following a 3-month telephone follow-up (T3). A repeated measures analysis of variance was used to determine group differences. Depression severity was dichotomized as minor (HAM-D, 11-14) and moderate-to-major depression (HAM-D, $\geq 15$ ), and group

intervention and control responses were also evaluated on that basis. Results: The greatest reduction in HAM-D scores over time occurred in the EX/CBT group (-10.4) followed by CBT (-9.6), EX (-7.3), and UC (-6.2), but none were statistically significant. The combined group showed a significant increase in 6-min walk distance at 24 weeks $(F=13.5, P<.001)$. Among all groups with moderate-to-major depression, only those in CBT/EX had sustained lower HAM-D scores at 12 and 24 weeks, 6MWT distances were significantly greater at $12(P=.018)$ and $24(P=.013)$ weeks, and the greatest improvement in HRQOL also occurred. Conclusions: Interventions designed to improve both physical and psychological symptoms may provide the best method for optimizing functioning and enhancing HRQOL in patients with HF.

Crown Copyright (C) 2010 Published by Elsevier Inc. All rights reserved.
\end{abstract}

Keywords: Cognitive behavioral therapy; Depression; Heart failure; Home-based exercise

\section{Introduction}

Depression is increasingly recognized as a strong predictor of health status and poor clinical outcomes in patients with heart failure (HF) $[1,2]$. Patients with HF have two to three times higher rates of depression than the general population and twice the rate of most other chronic medical illnesses $[3,4]$. Major depression is estimated to occur in

* Corresponding author. Nell Hodgson Woodruff School of Nursing, Emory University, Atlanta, GA 30322, USA. Tel.: +1 404727 0537; fax: +1 4047279382.

E-mail address: ragary@emory.edu (R.A. Gary). approximately $25 \%$ of $\mathrm{HF}$ patients; another $30 \%$ to $35 \%$ experience minor depression. Depressed HF patients experience a more rapid loss of physical function and onset of physical disability [5-8], greater symptom severity, poorer health-related quality of life (HRQOL), more frequent hospitalizations, and higher mortality rates than nondepressed HF patients [9-12]. Despite the deleterious consequences of depression in patients with HF, there are surprisingly few trials of treating depression in cardiovascular disease that has specifically targeted this population.

Depression increases both in prevalence and severity as $\mathrm{HF}$ worsens. For example, in a recent meta-analysis, Rutledge [13] reported that the rates of depression 
continued to increase nearly $10 \%$ per New York Heart Association (NYHA) class (11\% NYHA Class I up to $42 \%$ in NYHA Class IV), or a four-fold difference between Class I (asymptomatic) and Class IV (symptomatic at rest) patients. Numerous reports also suggest that depression increases morbidity and mortality in HF patients, independent of traditional cardiovascular risk factors [e.g., smoking, hypertension, low left ventricular ejection fraction (LVEF), high cholesterol and obesity] [2,13-15]. Similar underlying physiological mechanisms including hypothalamic pituitary axis, sympathoadrenal medullary, and immune responses are activated, bidirectionally and additively, when both HF and depression are present $[2,13]$. It is through these physiological pathways and behavioral mechanisms (sedentary, nonadherence, unhealthy lifestyle) that depression is thought to increase risk for adverse cardiac events and worsen clinical outcomes in HF patients. It is well established that HF patients who are depressed more often fail to adhere to prescribed medical regimens [16] and participate in high-risk behaviors such as smoking and alcohol use $[1,17,18]$; they also use more health care resources [19-21] and have poorer HRQOL [22-25] than nondepressed patients.

Considerable evidence supports that depression and physical function are mutually reinforcing and change in the same direction over time; when one worsens, the other also worsens [4]. Patients who are depressed perceive greater symptom severity, poorer physical function, and often curtail physical activity level to avoid worsening exertional intolerance and considerably lower HRQOL in patients with HF. Reduced physical activity enhances the deleterious peripheral and musculoskeletal pathological changes that are known to worsen HF symptom severity and accelerate disease progression $[2,6,8,13]$. An intervention that concomitantly maximizes physical function and fosters problem-focused coping, therefore, may provide the best opportunity for reducing depression, improving clinical outcomes, and enhancing HRQOL in patients with HF.

Treating depression is especially challenging in patients with HF because they are often older, have multiple comorbidities, and are on complex medication regimens $[2,13,26]$. Antidepressants often do not completely resolve depression in patients with HF, and relapse rates are high [27-29]. In addition, patients with HF who take antidepressants may be at higher risk for adverse events and hospitalization [26]. Moreover, the largest randomized study conducted to date in patients with HF experiencing depression, the Safety and Efficacy of Sertraline for Depression (SADHART) in Chronic Heart Failure [30], found that nurse-led control visits were as effective as antidepressant therapy for depression. For these reasons, nonpharmacological interventions for depression in HF may be particularly suitable [31,32]. Nonpharmacological interventions may provide important advantages over antidepressant therapy alone, including fewer drug interactions, greater short-term relief of depressive symptoms, and more involvement of patients in their self-care, but none have been reported in patients with HF [31-34].

Cognitive behavioral therapy (CBT) incorporates techniques such as self-monitoring, problem solving, and mutual goal setting that reinforce the benefits of exercise (EX) as well as other essential self-care strategies that improve clinical outcomes in patients with HF [35,36]. Coping skills can be taught using CBT strategies that attend to the physical and functional changes and consequences of HF, promote a positive health attitude, and facilitate adaptation, reducing the psychological burden [33-37]. Exercise holds promise for improving outcomes in HF, yet EX alone has not been shown to change the distorted thought patterns and negative perceptions that contribute to maladaptive coping in depressed HF patients. Exercise is known to partially reverse the underlying skeletal muscle changes that contribute to worsening $\mathrm{HF}$ and symptom severity [38-46] and has also been shown to increase physical function and improve HRQOL in a number of studies, but it has not been specifically used to alleviate depression in patients with HF [13].

The purpose of this randomized, controlled, repeated measures study was to compare the efficacy of three interventions (a combined 12-week home-based EX/CBT program, CBT alone, and EX alone with usual care (UC) in stable, NYHA II to III HF patients with minor or major depression at baseline (BL) according to Diagnostic and Statistical Manual-IV (DSM-IV) criteria [47]. We hypothesized that patients in the combined EX/CBT group would have a greater reduction in depression severity and better physical function and HRQOL compared to the other groups at 12 and 24 weeks.

\section{Methods}

Men and women between the ages of 30 and 70 who were an outpatient in an HF clinic in Northeast Georgia with a documented diagnosis of NYHA Class II to III heart failure and a Beck Depression Inventory II (BDI-II) [48] score of 10 or higher were asked to participate in the study. Participant inclusion criteria included (a) documented medical diagnosis of HF; (b) LVEF of $\geq 15 \%$ documented within the last year by echocardiogram, cardiac catheterization ventriculography, or radionuclide ventriculography; (c) receiving therapy for HF according to guidelines published by the American College of Cardiology American Heart recommendations [49] (angiotensin-converting enzyme inhibitors, diuretics, beta blockers, angiotensin receptor blockers, hydralazine and nitrate combination, etc.); (d) Hamilton Rating Scale for Depression (HAM-D) score $\geq 11$ [50]; (e) positive results on the Mini International Neuropsychiatric Interview (MINI) [51,52] for minor or major depression; and (e) DSM-IV diagnosis [47] for depression for 14 days; or 7 days if history of major depressive disorder (MDD) in the last 6 months. Participants also had to be (a) English 
speaking, (b) living independently (noninstitutionalized) within 100 miles of Atlanta, GA, (c) able to respond to questions appropriately, (d) able to hear adequately to respond to verbal questions, (e) not involved in any structured EX program or walking three times per week for a minimum of $20 \mathrm{~min}$, (f) not participating in any psychotherapy, and $(\mathrm{g})$ not hospitalized within the last 60 days. Exclusion criteria included (a) suicide ideation according to psychiatric assessment or MINI [51,52] evaluation; (b) major psychiatric comorbidity such as schizophrenia, personality disorder, or dementia; (c) planned surgery; (d) not diagnosed with HF in the past 3 months; (e) renal insufficiency (serum creatinine $>2.5 \mathrm{mg} / \mathrm{dL}$ ); (f) uncontrolled hypertension; ( $\mathrm{g}$ ) acute bereavement or loss of significant other within the last month or currently involved in family crisis such as divorce; (h) any disorder interfering with independent ambulation; and (i) terminal illness such as cancer.

\section{Outcome measures}

Depression was evaluated using the Hamilton Rating Depression Scale (HAM-D) [50], a 17-item clinical rating scale, and the MINI [51,52]. The MINI was only used at BL and only to ensure diagnostic precision for outcome tracking. The HAM-D was used to record depression severity at BL and track depression over time. A score of 8 or below on the HAM-D was considered clinical remission. The HAM-D was administered in a standardized, semistructured format by a trained data collector. Interrater reliability was established at 0.90 or above between two data collectors using mock sessions prior to enrolling patients.

Physical function was indirectly assessed using the 6-min walk test (6MWT) [53]. The 6MWT is a frequently used, reliable, and well-validated measure of physical function in HF patients. Construct validity was observed by correlating the 6MWT with HRQOL [Minnesota Living with Heart Failure Questionnaire (MLHFQ)] $(P=.0001)$ and the NYHA functional class $(P=.001)$ in 768 patients enrolled in the Randomized Evaluation of Strategies for Left Ventricular Dysfunction pilot study [54-56]. Studies have also shown that the 6MWT is lower in some patients who are depressed compared to those who are not depressed [57].

Health-related quality of life was evaluated using the MLHFQ [58]. The MLHFQ is a well-established, 21-item, disease-specific questionnaire designed to measure perceived physical, socioeconomic, and psychological impairment of persons with HF. Scores range from 0 to 105, with higher scores indicating of poorer HRQOL. A change in score of 5 points or more is considered clinically significant for improvement in symptom severity and HRQOL. The MLHFQ is more sensitive to change over time than other global measures [58]. Cronbach's $\alpha$ reliabilities of the total MLHFQ, the physical subscale, and the emotional subscale are reported at $.87, .81$, and .84 , respectively, and are consistently above .70 in published reports [59].

\section{Procedures}

After written informed consent was obtained using the affiliated (Emory University) Institutional Review Board guidelines, patients were initially screened for depressive symptoms using the BDI-II [48]. Potential study candidates with a BDI-II [52] score of 10 or higher were further screened for minor and major depression using the Mini Neuropsychiatric Interview (MINI) [51,52] and the HAM-D [50]. Depression severity was evaluated for patient safety reasons using the HAM-D at 5 time points (BL, 4, 8, 12, and 24 weeks), which was guided by a treatment algorithm described below.

Data collectors were blinded to group assignment. Psychiatric clinical nurse specialists or clinical psychology doctoral students administered the CBT intervention; all received additional training in Beck's $\mathrm{CBT}$ and participated in mock patient sessions prior to enrolling participants described in detail below. All patient therapy sessions were conducted at home, audiotaped; each interventionist received ongoing feedback regarding adherence to CBT principles using the cognitive therapy rating scale (CTRS) provided by the Beck Institute for Cognitive Therapy and research (www. beckinstitute.org) [60]. Participants in the combined EX/ CBT or EX only group were assigned a nurse interventionist for patient safety reasons.

After BL measures were taken, patients were randomized to one of four groups described below. Home visits were made within 2 weeks following randomization for the intervention groups. After the 12-week intervention or control condition, T2 measures were taken at the physical function laboratory, and intervention participants received telephone calls weekly, then bimonthly, and at the end of 3 months to monitor walking progress and or depressive symptoms. Those in the CBT and combined groups continued with CBT telephonic counseling. Participants in the EX only group were provided ongoing tailored EX advice based on their feedback. At the end of the 3-month follow-up period, T3 measures were again taken at the physical function laboratory.

\section{Intervention groups}

\section{Exercise therapy}

Participants assigned to the EX group had 12 weekly faceto-face home visits to monitor walking progress and to tailor the EX prescription. At the first home visit for EX, the research nurse (1) educated the patient on the rationale for EX in HF; (2) instructed on self-monitoring of symptoms [dyspnea, heart rate (HR), fatigue] during walking; (3) provided the patient with a Polar monitor and instruction on how to use it; (4) provided patient with EX logs and instructions; (5) instructed on use of the 6- to 20-point Borg's rate of perceived exertion (RPE) scale [61]; (6) provided patient with blood pressure cuff and weight scale, if not available; and (7) observed participant response to walking 
outside the home. Initially, participants were advised to walk 3 days per week for 12 weeks. As EX tolerance increased, participants were advised to increase duration by $5 \mathrm{~min}$ weekly to a maximum of $1 \mathrm{~h}$ for 3 days per week at moderate intensity. The goal of the walking program was to have the EX participants walking $20 \%$ above BL intensity over the 12-week period. Polar heart monitors [62] assessed HR during the intervention, and the RPE scale was used to monitor exertion level. Patients were instructed to keep their RPE below 15 and HR within the prescribed range based on the HR reserve method [63]. Participants' recorded time and duration of each walking session, HR, blood pressure, weight, and symptoms in weekly walking logs. If the surrounding home environment was not conducive to walking, or if there was adverse weather that prohibited outside walking, the participant was encouraged to EX at the closest venue (e.g., large grocery store, high school gym). Adherence rates were calculated based on documentation of walking in the logs. Sessions lasted between 30 and $45 \mathrm{~min}$ depending upon patient tolerance level.

The CBT intervention was based on Beck's CBT model of depression [35,36]. Beck's CBT postulates that depressed individuals hold negative mental representations of themselves, their world, and their future. These views are activated by negative life events (e.g., HF), leading to negatively biased thinking and interpretation of situations. In contrast to traditional, long-term psychoanalytic therapy, CBT for depression is structured, collaborative, short term, and problem focused; it teaches patients a variety of cognitive and behavioral skills that can be employed to combat depression. The first two to three sessions were used to (1) build and establish rapport with the patient, (2) review principles of the cognitive model (agenda, thoughts, influence, behavior), (3) educate the patient about depression (negative schema), (4) teach the patient about CBT methods that may be used (i.e., identifying automatic thoughts, activity scheduling, role playing, homework), (5) establish mutual collaborative goals for therapy, and (6) clarify concerns and answer any questions about CBT. Depressive symptoms were monitored weekly using moodrating charts, which was simple and successful in capturing any changes $[35,36,64]$. Each session lasted approximately $1 \mathrm{~h}$ in duration.

\section{Combined CBT/EX}

Patients assigned to this intervention received a combination of the weekly walking and CBT sessions as described above. One interventionist delivered both the walking and CBT program either concurrently or separately depending on patient tolerance.

\section{Usual care group}

Participants assigned to the UC group received no information or counseling from their health care provider other than that normally provided. The UC group had the same schedule of measures, including the five HAM-D measures administered at $\mathrm{BL}, 4,8,12$, and 24 weeks, and payment incentives as those in the intervention groups, but they received no home visits during the study period.

\section{Depression treatment algorithm}

A depression treatment algorithm based on the American Psychiatric Association guidelines for treatment of depression [65] was implemented for all study participants to reduce the risk for adverse events. Mood scores were collected weekly during the 12 home visits and by telephone during the 3-month follow-up period to monitor depressive symptoms. For the control participants, mood checks were conducted during the time scheduled measurements were taken. In addition, control participants were provided with contact information for the research team should they have an increase in depressive symptoms. If there were sudden changes and suicide ideation or $>50 \%$ increase in weekly mood scores occurs, the study psychiatrist was contacted immediately for treatment evaluation and the patient excluded from further study participation. Otherwise, if there were no acute changes, the treatment plan was evaluated at 8 weeks and again at 12 weeks.

A successful outcome from the intervention was defined as a $50 \%$ reduction in HAM-D scores and two consecutive weeks of HAM-D scores $\leq 8$ at 12 (T2) and at 24 weeks (T3). If the participant had a HAM-D score of $\leq 8$ prior to the conclusion of the 12-week intervention, treatment was continued for the allotted time period in order to achieve medium and long-term goals and to reduce the risk of depression relapse, defined as the return of depression symptoms or dysfunction following remission [65]. We monitored medication changes and additions across all four groups. If antidepressant medication was prescribed, the participants were monitored for drug response by their research nurse. If a participant remained depressed at the conclusion of the 12-week intervention, he or she was referred to the study psychiatrist for further evaluation and treatment. If a participant had relapsed or had higher scores on the HAM-D scale or increased depression, as measured by mood ratings during telephone follow-up sessions or at $\mathrm{T} 3$, the study psychiatrist was contacted for further advisement.

\section{Training and intervention fidelity}

Protocol manuals outlined the goals and objectives and a plan for each CBT session and the roles of the interventionists. Cognitive behavioral therapy training was conducted by a psychologist trained in CBT and included didactic information about depression, cognitive processes, and CBT strategies. We used interventionist-to-interventionist and mock patients to demonstrate CBT strategies during training. Role playing, audiotaping, and videotaping mock intervention sessions (interventionist-to-interventionist and nurse-to-mock patients) were conducted prior to patient enrollment. Each interventionist recorded all sessions with patients and turned in weekly audiotapes to the 
CBT supervisor. The CTRS was used to assess both the strengths and weaknesses in the audiotaped CBT sessions and to provide specific feedback for each of the interventionists [60].

Initially, the CBT supervisor met weekly with the interventionists to review principles of $\mathrm{CBT}$ and discuss specific examples of patient responses (considered part of CBT training). After the first 12 to 16 weeks, biweekly and then monthly supervision meetings were held. Three audiotaped sessions were evaluated by the CBT supervisor at randomly selected times to reflect early, mid, and late therapy sessions.

Exercise supervision was provided by the principal investigator and was conducted in participants' homes. An EX fidelity checklist was used for this purpose. The same interventionists who delivered the CBT sessions also delivered the EX intervention for the combined group. Each nurse received instructions on self-monitoring techniques, use of the Polar HR monitor, and ways to help patients progress in relation to the duration, frequency, and intensity of EX according to the American Heart Association and Heart Failure Society of America [39].

\section{Data analysis}

Descriptive statistics and repeated measures analysis of variance (RMANOVA) were used to describe sample characteristics and group differences, respectively. Covariates adjusted for the RMANOVA analysis included age, gender, NYHA class, and antidepressant use. After the entire participant pool was examined collectively for group differences, we then dichotomized depression severity as minor (HAM-D, 11-14) and moderate-to-major depression (HAM-D, $\geq 15)$ and evaluated group intervention and control responses on that basis. Statistical analyses were carried out using the Statistical Package for Social Science (SPSS/-PC+), 16.0 version (SPSS, Chicago, IL). A level of significance of $P<.05$ was used for hypotheses testing.

\section{Participants}

Over 14 months, 982 patients were referred, responded to advertisements, or were contacted by letter about the study at four hospital sites. Of these, 242 met eligibility criteria for NYHA Class II or III HF (systolic or diastolic) and were screened for depression using the BDI-II. Of the 242, 137 had a BDI-II score of 10 or higher and received MINI and HAM-D screening. Of this number, 74 participants met eligibility criteria of minor or major depression and were consented for study participation (Fig. 1). Reasons for not participating in the study included time constraints, lack of interest, did not think they were depressed, or did not want to discuss their feelings or private lives with others. Participant characteristics are presented in Table 1. After BL measures were taken, participants were randomized to UC $(n=17)$, EX $(n=20)$,
CBT $(n=19)$, or combined EX/CBT $(n=18)$. There were no BL differences between groups on any demographic or outcome variables. The $77 \%$ majority $(n=57)$ reported a prior episode of MDD, and only $29 \%(n=22)$ were currently prescribed antidepressants, though all met $D S M-I V$ criteria for depression. There were no differences in the number of patients receiving antidepressant therapy at BL; no patients were started on antidepressant medication, nor were any antidepressant dosages changed during the 6-month study period. At BL, scores on the HAM-D (11-14) indicated that $39 \%(n=29)$ had minor depression and 60\% $(n=44)$ moderate-to-major depression (HAM-D, $\geq 15$ ). Three withdrew from CBT only, three from EX, three from EX/ CBT, and three from UC. Two patients, both in the 3-month follow-up phase, died while enrolled (one in CBT, one in EX), from causes not related to the study. One participant was excluded from data analysis due to an abnormally high HAM-D score, which artificially raised group results. There were no BL differences between those who withdrew or continued in the study.

\section{Results}

\section{Depression outcome}

Examining the results for the entire participant pool, there were declines in HAM-D scores over 5 time points (BL, 4, 8, 12, and 24 weeks) in all four groups (Fig. 2), but none were statistically significant. Notably, when compared to the other groups, only the combined EX/CBT group had a sustained reduction in depression at 12 and 24 weeks. However, data analysis revealed that when participants were dichotomized as having minor depression (HAM-D scores between 11 and 14 ) or moderate-to-major depression (HAM-D scores, $\geq 15$ ), there were considerable differences in the patterns of the study's findings on its major outcome variables (depression, physical function, and HRQOL). Therefore, the findings were also dichotomized into minor and moderate-to-major depression and presented below for greater clarity (Table 2; Figs. 3 and 4).

The key finding related to the depression outcome for those with minor depression was at 24 weeks; clinical remission (HAM-D $<8$ ) occurred in all groups, including UC (Fig. 3). HAM-D decline in the CBT only group was greater than the other groups from 8 to 24 weeks. Once the CBT only group achieved remission at 8 weeks, they remained in remission for the entire 24-week duration of the study. Although the EX only, UC, and combined groups had higher depression symptoms at 12 weeks, all groups were in remission at 24 weeks.

Only the CBT/EX group had sustained lower HAM-D scores for those with moderate-to-major depression at both 12 and 24 weeks as shown in Fig. 4. The CBT only group had the lowest HAM-D scores for those with moderate-tomajor depression at 8 weeks, but this reduction was not 


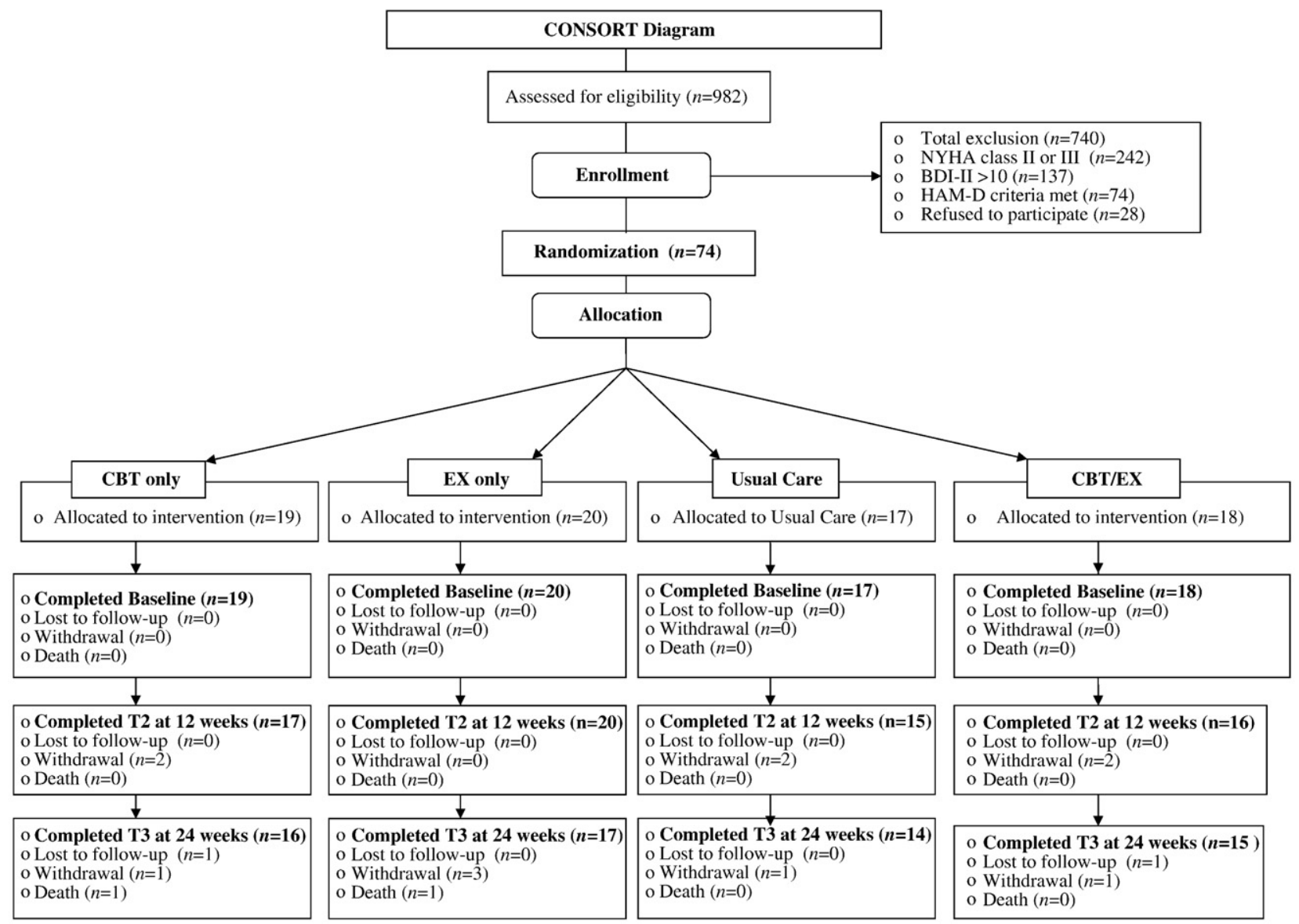

Fig. 1. Consort flowchart.

Table 1

Baseline sociodemographic and clinical characteristics $(n=74)$

\begin{tabular}{ll}
\hline Characteristic & Total \\
\hline Age $(M \pm$ S.D. $)$ & $65.8 \pm 13.5$ \\
Gender $(n, \%)$ & \\
Male & $31(41.9)$ \\
Female & $43(57.1)$ \\
Ethnicity $(n, \%)$ & \\
Caucasian & $53(71.6)$ \\
African American & $21(28.4)$ \\
Education $(n, \%)$ & \\
High school & $42(56.8)$ \\
Tech/college & $23(31.1)$ \\
Marital status $(n, \%)$ & \\
Married & $29(39.2)$ \\
Widowed & $21(28.4)$ \\
Divorced/single & $23(31.1)$ \\
NYHA Class $(n, \%)$ & \\
II & $32(43.3)$ \\
III & $42(56.7)$ \\
Comorbidity $(M \pm$ S.D.; range) & $2.9 \pm 1.7 ; 1-8$ \\
Hypertension $(n, \%)$ & $60(88.2)$ \\
Myocardial infarction $(n, \%)$ & $23(34.4)$ \\
Diabetes $(n, \%)$ & $22(32.4)$ \\
Major depression history $(n, \%)$ & $52(77.0)$ \\
Total Medications $(M \pm$ S.D.; range) & $7.8 \pm 3.9 ; 2-20$ \\
Antidepressants $(n, \%)$ & $22(29.7)$ \\
Antianxiety $(n, \%)$ & $9(12.2)$ \\
\hline
\end{tabular}

sustained at 12 and 24 weeks. Both the EX and UC groups began an increase in depressive symptoms at 12 and 24 weeks.

When efficacy for reducing depression severity from moderate-to-major depression to minor depression was compared among groups, only the CBT/EX group showed significant differences for lowering moderate-to-major depression to minor depression at $8(P=.049), 12(P=.001)$, and 24 weeks $(P=.014)$.

\section{Physical function outcome}

Examining the results for the entire participant pool, the combined group showed significantly greater improvement in 6-min walk distance at 24 weeks $(F=6.9, P=.001)$ compared with the other groups. In the CBT and UC groups, there was a considerable and progressive decline in 6MWT as shown in Fig. 5. The only group that improved and showed positive increases in physical function at both time points was the EX/CBT group.

When the participant pool was dichotomized, the impact of the combined intervention on those with moderate-tomajor depression is much stronger. Among all groups with moderate-to-major depression, only those in EX/CBT had $6 \mathrm{MWT}$ distances that were significantly greater than BL at 


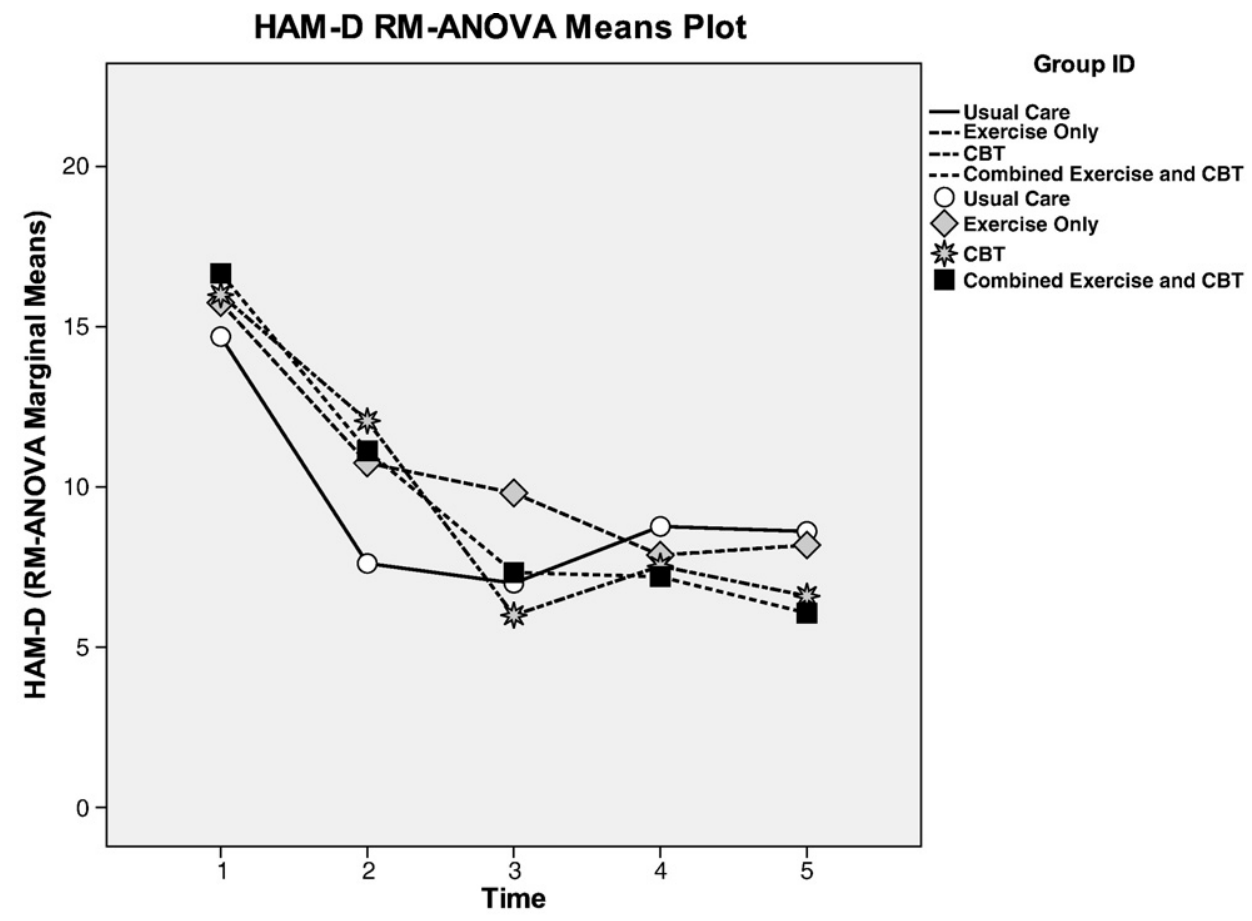

Fig. 2. HAM-D responses for minor and moderate-to-major depression by group.

$12(P=.018)$ and $24(P=.013)$ weeks as shown in Fig. 5. For the total sample, the effect size was 0.485 , a moderateto-large effect size. However, when the EX/CBT group was compared to the other groups for the 6MWT among those with moderate to major depression we found a large effect size (0.531). The distance walked on the 6MWT at BL was lower in patients with MDD compared to MinD (282.7 \pm 138.5 vs. $334.9 \pm 120.7$, respectively).

\section{HRQOL outcome}

Examining the entire subject pool, the EX/CBT group had the greatest improvement in MLHFQ scores over the duration of the study, as shown in Fig. 6, but none reached statistical significance. The EX/CBT group had a 10.1-point drop in scores from T1 to T3 (lower scores indicate better perceived HRQOL); this is a clinically meaningful change (greater than 5 points) (Table 3). Of note, the CBT group had the most improved HRQOL from BL to T2. However, this improvement was not sustained; at 24 weeks, this group had lower MLHFQ scores than the other groups, but it was not statistically significant.

Once again, a more detailed analysis demonstrates greater efficacy for CBT/EX among those with moderate-to-major depression. As Fig. 6 illustrates, CBT/EX participants with moderate-to-major depression demonstrated greater improvement in HRQOL at 12 and 24 weeks (lower scores) than those in the other groups.

Table 2

Depression screening and depression outcome variables at BL, 4, 8, 12, and 24 weeks

\begin{tabular}{|c|c|c|c|c|c|c|}
\hline & $\mathrm{BL}$ & 4 weeks & 8 weeks & 12 weeks & 24 weeks & $P$ value* \\
\hline \multicolumn{7}{|l|}{ BDI-II } \\
\hline Total group & $20.7 \pm 7.5(74)$ & & & & & \\
\hline CBT only & $20.6 \pm 9.4(19)$ & & & & & \\
\hline EX only & $19.5 \pm 4.8(20)$ & & & & & \\
\hline $\mathrm{UC}$ & $20.7 \pm 7.5(17)$ & & & & & \\
\hline CBT/EX & $22.0 \pm 8.5(18)$ & & & & & \\
\hline \multicolumn{7}{|l|}{ HAM-D } \\
\hline Total group & $15.9 \pm 4.3(74)$ & $10.85 \pm 5.3(73)$ & $7.9 \pm 5.1(67)$ & $8.3 \pm 5.2(66)$ & $7.4 \pm 5.1(62)$ & NS \\
\hline CBT only & $15.9 \pm 4.9$ (19) & $11.3 \pm 3.9(19)$ & $6.9 \pm 4.9$ (18) & $8.2 \pm 6.3(17)$ & $7.1 \pm 4.9(16)$ & NS \\
\hline EX only & $15.4 \pm 3.4(20)$ & $10.4 \pm 3.9(20)$ & $9.8 \pm 5.7(18)$ & $8.4 \pm 5.6(18)$ & $8.3 \pm 5.2(17)$ & NS \\
\hline $\mathrm{UC}$ & $15.2 \pm 3.6$ & $13.8 \pm 5.2(17)$ & $11.0 \pm 4.9(15)$ & $9.3 \pm 4.9(15)$ & $8.2 \pm 5.4$ (14) & NS \\
\hline $\mathrm{CBT} / \mathrm{EX}$ & $17.1 \pm 4.3(18)$ & $10.8 \pm 5.8(17)$ & $7.4 \pm 4.7(16)$ & $6.5 \pm 3.7(16)$ & $6.1 \pm 5.1(15)$ & NS \\
\hline
\end{tabular}

* $P$ values reflect RMANOVA across all time points controlling for age, gender, NYHA class, and antidepressant use. 


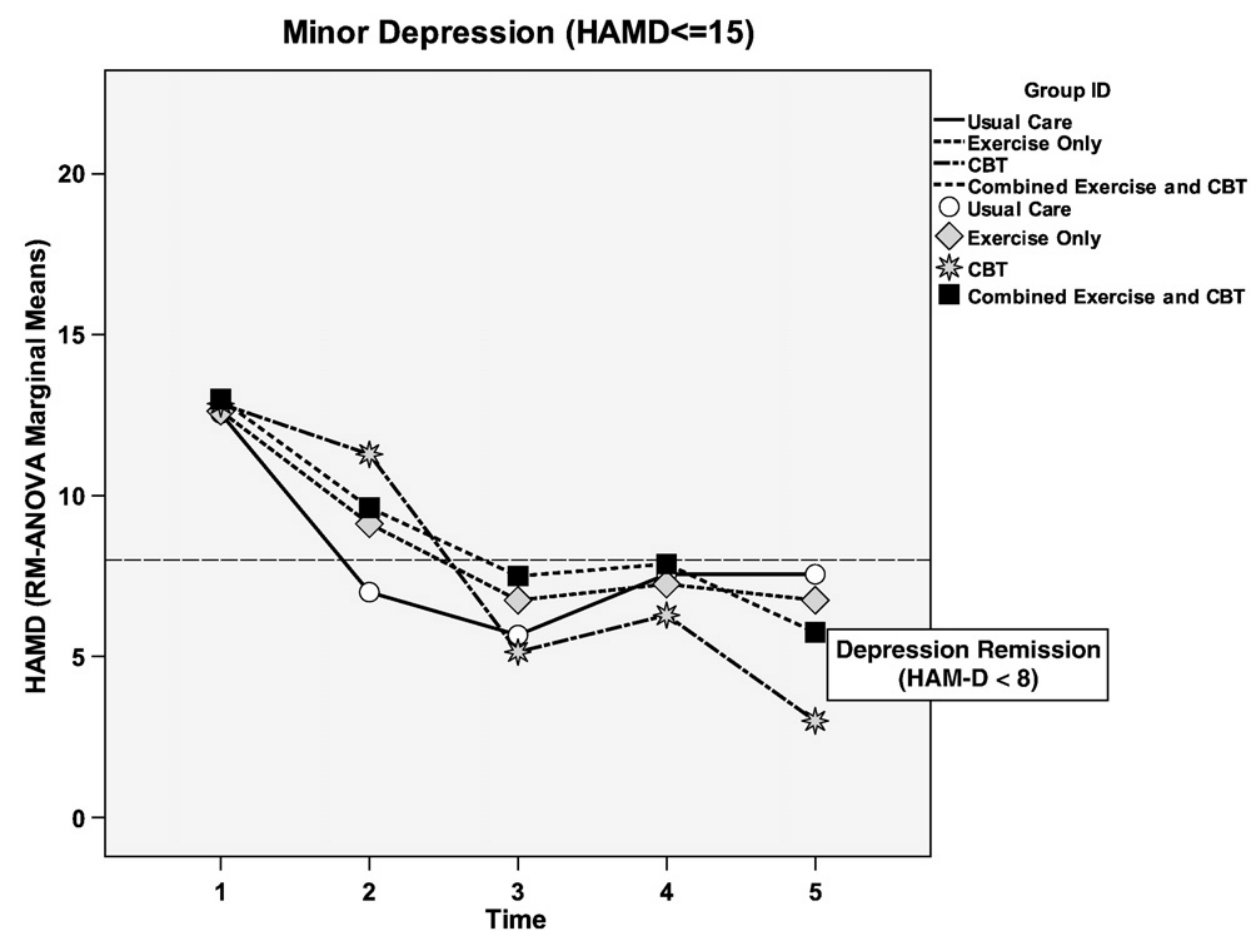

Fig. 3. HAM-D scores across all groups for minor depression.

Adherence scores were calculated based on the number of sessions attended of those prescribed. In the EX group, a walking $\log$ record indicating walking a minimum of three times per week at the prescribed duration was considered $100 \%$ adherent. In the CBT group, $100 \%$ adherence was defined as participation in all weekly CBT sessions. In the combined group, half of $100 \%$ adherence was defined as walking three times per week for a minimum of $20 \mathrm{~min}$, and the other half was defined as participation in all weekly CBT sessions. For each intervention group, weekly adherence rates were recorded and divided by the number of weeks in $\mathrm{EX}, \mathrm{CBT}$, or the combined program for a mean adherence

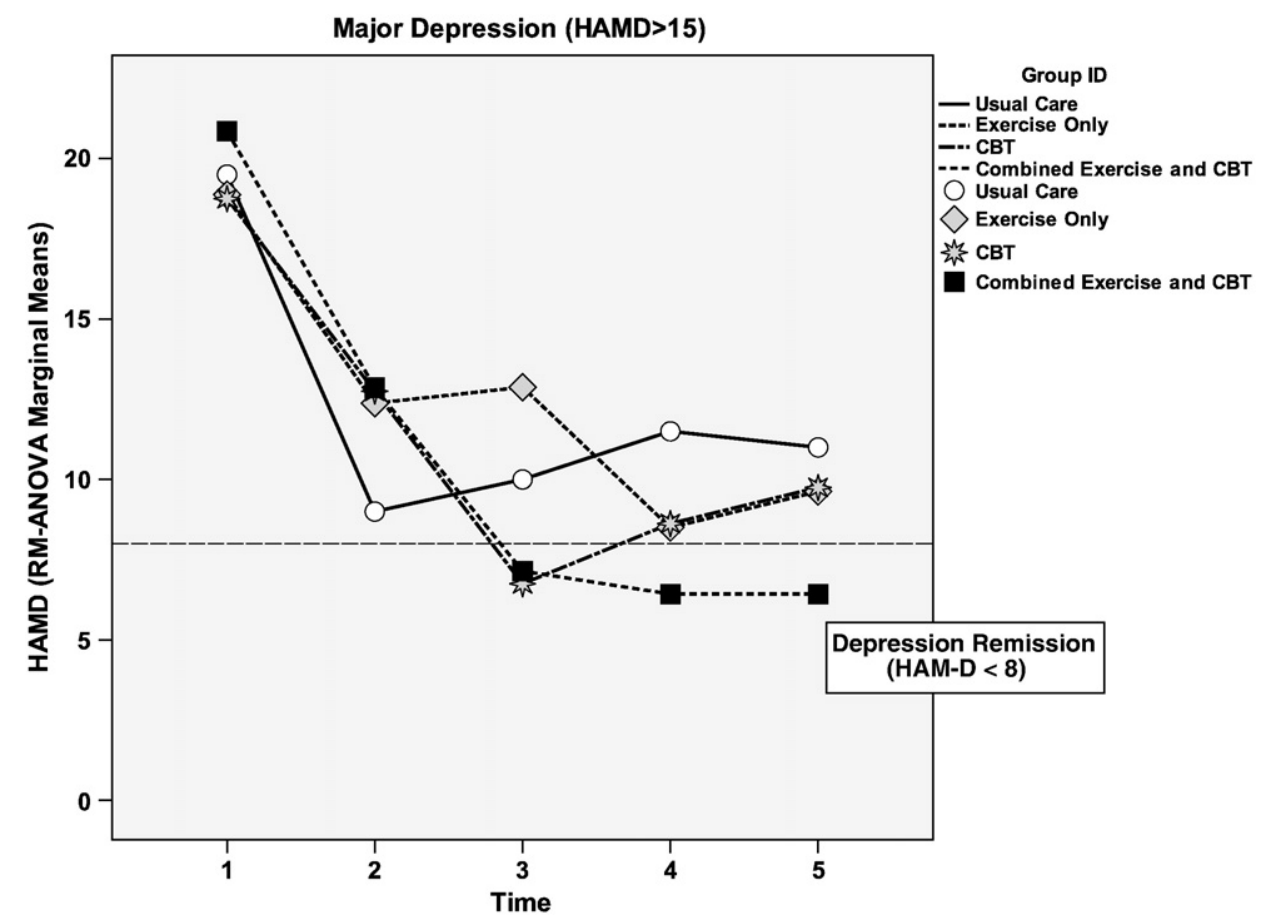

Fig. 4. HAM-D across all groups for moderate-to-major depression levels. 


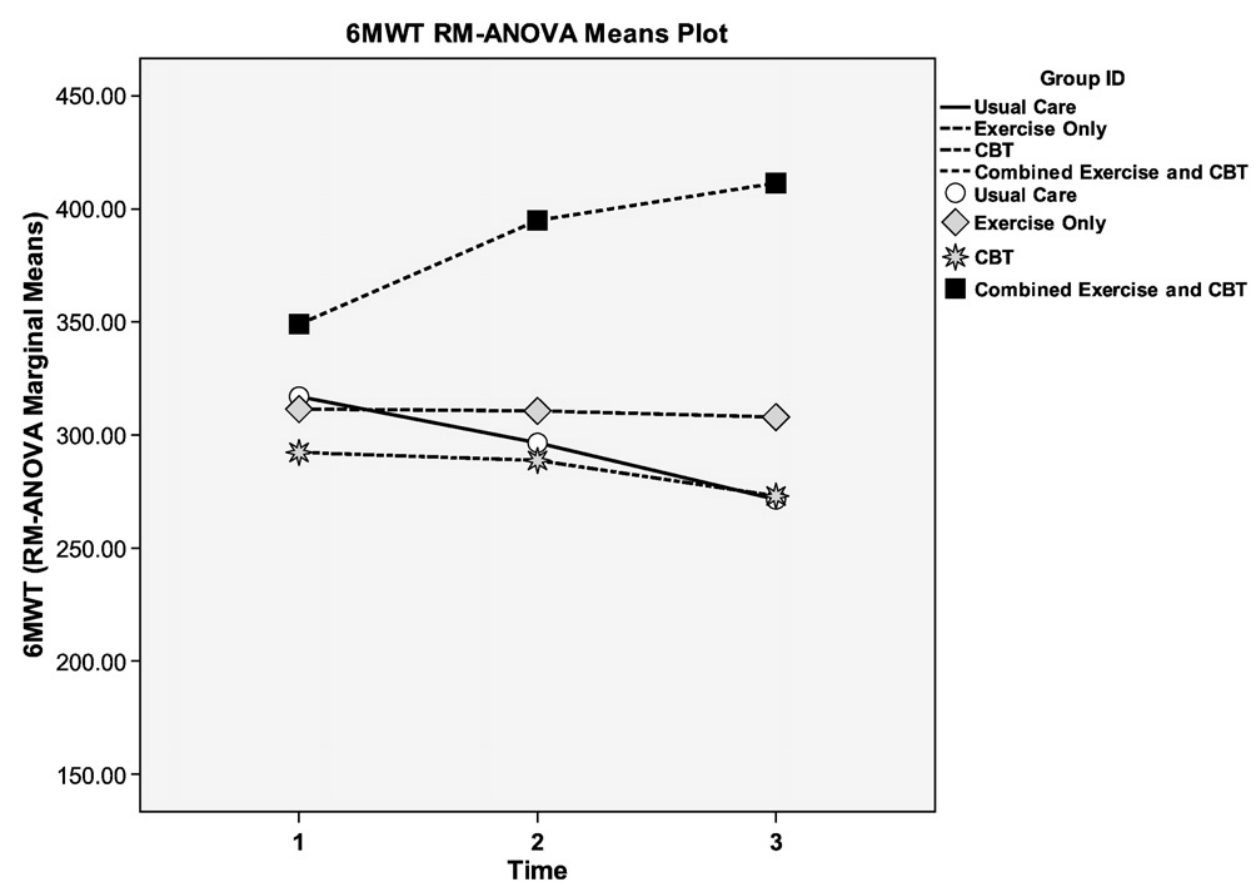

Fig. 5. 6MWT for all groups for minor and moderate-to-major depression.

score. The highest adherence rates were reported in the EX/ CBT group (85\%) followed by EX (82\%) and CBT (72\%). Reasons for missing sessions were the same across groups and included scheduling conflicts, hospitalization, and not feeling well. No related adverse events were reported during the study.

\section{Discussion}

The combined EX/CBT group was the only group that showed sustained improvement on all primary outcome variables at 12 and 24 weeks, and that the intervention effects were stronger in patients with moderate-to-major depression (HAM-D 15 or higher). All patients who had minor depression (HAM-D, 11-14) improved over time despite group assignment, indicating that intervention may not be necessary in these patients. Because $40 \%$ of our patients had minor depression and the small sample size in each group, we think the potential efficacy of the combined intervention may have been diminished. A weakened intervention effect has also been shown in recent trials of antidepressant therapies due to the inclusion of patients with

RM-ANOVA QOL - Moderate-to-Major Depression (HAMD-baseline >= 15)

Moderate-to-Major vs Minor Depression: Moderate-to-Major Depression (15 and higher)

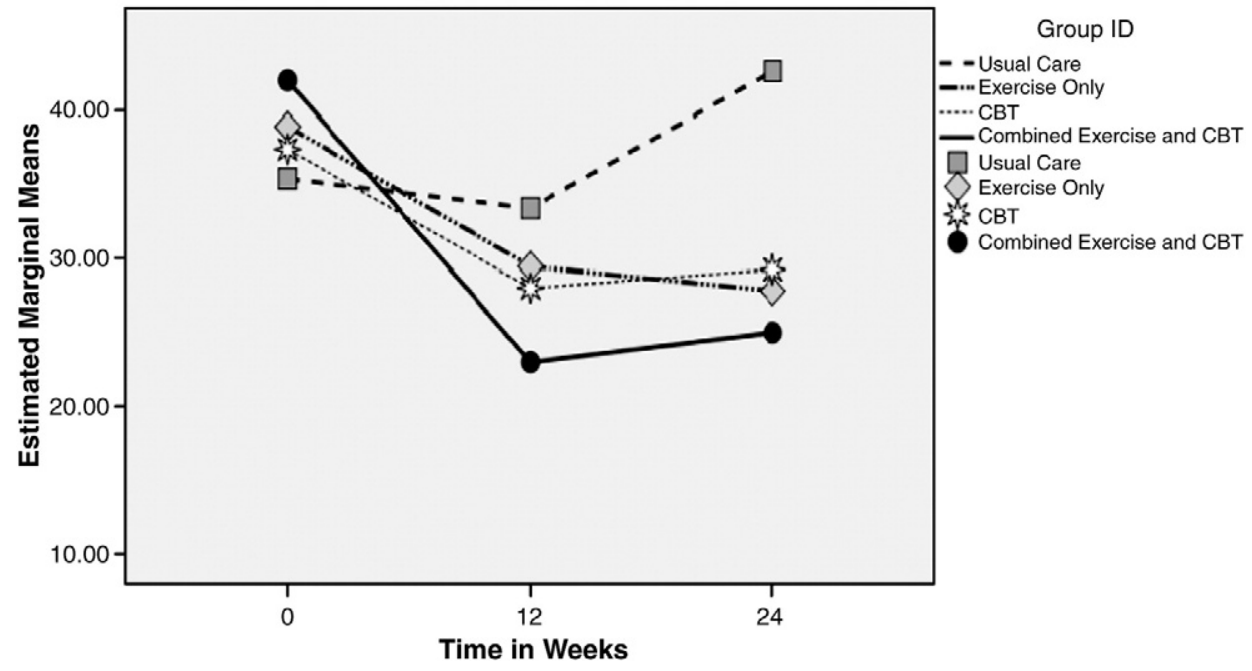

Fig. 6. Baseline HRQOL scores at BL, 12 and 24 weeks for HAM $>15$. 
Table 3

Change in HRQOL and 6MWT outcome variables at BL and 12 and 24 weeks

\begin{tabular}{|c|c|c|c|c|}
\hline & BL & 12 weeks & 24 weeks & $P$ value * \\
\hline \multicolumn{5}{|l|}{ MLHFQ } \\
\hline Total group & $34.5 \pm 23.0(74)$ & $27.5 \pm 23.1(66)$ & $28.1 \pm 22.5(62)$ & NS \\
\hline CBT only & $35.3 \pm 27.2$ (19) & $26.7 \pm 20.2$ & $34.3 \pm 23.6(16)$ & NS \\
\hline EX only & $32.4 \pm 22.4$ (20) & $29.2 \pm 18.1(20)$ & $25.6 \pm 19.7(17)$ & NS \\
\hline $\mathrm{UC}$ & $28.1 \pm 17.3(17)$ & $26.4 \pm 23.7(15)$ & $28.9 \pm 29.9(14)$ & NS \\
\hline $\mathrm{CBT} / \mathrm{EX}$ & $34.3 \pm 20.2(18)$ & $27.3 \pm 16.8$ & $24.2 \pm 16.3(15)$ & NS \\
\hline \multicolumn{5}{|l|}{ 6MWT } \\
\hline Total group & $313.5 \pm 130.0(74)$ & $321.5 \pm 128.4(66)$ & $315.0 \pm 139.1(62)$ & NS \\
\hline CBT only & $335.4 \pm 125.3$ (19) & $290.9 \pm 134.8(17)$ & $317.9 \pm 132.3(16)$ & NS \\
\hline EX only & $326.3 \pm 135.8(20)$ & $307.8 \pm 125.3(20)$ & $317.9 \pm 132.3(17)$ & NS \\
\hline $\mathrm{UC}$ & $347.3 \pm 166.2(17)$ & $287.1 \pm 125.3(15)$ & $271.5 \pm 161.7$ (14) & NS \\
\hline $\mathrm{CBT} / \mathrm{EX}$ & $363.1 \pm 100.2(18)$ & $400.6 \pm 102.5(16)$ & $411.5 \pm 100.8(15)$ & .001 \\
\hline
\end{tabular}

* $P$ values reflect RMANOVA across all time points controlling for age, gender, NYHA class, and antidepressant use; NS=nonsignificant.

minor depression (e.g., SADHART) [66]. We note that patients who were taking antidepressants had no better outcomes than those who were not taking them at BL, and no additional pharmacological therapy was required during the study period, indicating that the interventions were efficacious in reducing depressive symptoms, especially among those with moderate-to-major depression. Although some patients in the control group did not reduce depressive symptoms, none increased to the point where additional intervention was necessary. These findings also suggest that booster sessions between 12 and 24 weeks may have prevented depression relapse since many patients began to have elevated depression scores during this period. Other studies show that booster sessions are effective for preventing relapse [67].

To our knowledge, this pilot study is one of the largest nonpharmacological interventions conducted to date to attempt to reduce depression in patients with HF. A very small limited study evaluating the impact of CBT in 22 NYHA Class II or III HF patients found that the intervention group (group-based CBT plus EX) experienced a $52 \%$ reduction in depressive symptoms, while the other groups (titrated Digoxin or placebo) experienced a $15 \%$ and $25 \%$ increase in depressive symptoms, respectively. However, the design of the study made it difficult to determine which treatment (EX or CBT) was most beneficial in lowering depressive symptoms [68]. Our findings support the superiority of a combined regimen of CBT and EX over therapy alone. Further studies are needed in a more diverse and adequately powered sample of HF patients to determine which therapy either alone or in combination may be most effective and feasible in clinical practice settings.

The finding that CBT only patients showed a significant decline in 6-min walk distance, similar to UC, was surprising, despite having significantly improved depressive symptoms. This suggests that verbally encouraging patients with HF to be physically active may not be sufficient to improve or maintain physical function. Patients in the CBT only and UC groups were instructed to continue their routine daily activities, and suggestions for activities were provided to reduce inertia and increase physical activity level, consistent with Beck's CBT. Patients in the EX only group, who did not receive $\mathrm{CBT}$, also showed little change in physical function at 12 and 24 weeks, indicating that motivation and adherence to the prescribed EX regimen may have been problematic for these patients, especially early in the intervention when depression rates may have been higher.

The relationship between physical function and depression severity in patients with $\mathrm{HF}$ has not been widely studied. The findings from this study suggest that higher depression negatively influences physical function and strongly supports the need for an intervention that addresses both psychological and physical function in patients with HF experiencing moderate-to-major depression. Studies have shown that using objective methods to track physical activity such as accelerometers or pedometers in patients with HF may be useful to ensure that the walking dose is adequate and to reinforce the beneficial effects of EX. Whether providing self-monitoring devices such as pedometers in patients with HF and depressed increases physical activity level either with or without explicit EX prescriptions is an area for future investigation.

The reason for the poorer HRQOL outcomes at 24 weeks in the CBT only group is unclear. We think that weekly faceto-face contact and the perceived social support provided during the 12-week intervention period may have contributed to improvement in HRQOL among the CBT group. This was particularly true among those who were elderly, who lived alone, who were homebound, and who resided in rural locations. For several of these participants, the therapy session was the only face-to-face contact during the week. The degree to which increased social support may have confounded the effect of the intervention was not thoroughly examined in this study. However, the fact that all intervention group participants essentially received the same weekly contact (hourly home visits for 12 weeks) and the controls received no visits suggests that the increased social interaction alone did not contribute to improved depression outcomes. Future trials should control for the 
effects of social support on depression outcomes to further tease out the differences between changes due to therapy vs. social interaction.

The majority of research on the efficacy of EX in depression has included small studies that lacked controls or adequate diagnostic measures of depression; thus, whether patients were experiencing depression or depressive symptoms in previous studies is unclear [69-72]. In addition, few prior studies controlled for the effects of social support in group EX, which is known to positively influence depressive symptoms through social interaction. By using a home-based approach to EX, we think the effects of group interaction were minimized. Furthermore, home-based EX programs in chronically ill, depressed patients and the dose of EX required to reduce depressive symptoms have also received little attention [71-73]. The findings from the current study suggest that home-based walking may be beneficial in depressed HF patients and may provide incentive for participation particularly among those who lack transportation or are homebound. Blumenthal et al. [73] found remission rates for elderly patients experiencing major depression randomized to receive a home-based EX intervention or medication (sertraline) were similar. Cardiac patients enrolled in cardiac rehabilitation and followed over a 5 -year period found that the prevalence of depressive symptoms decreased from $17 \%$ to $6 \%$, but again, standard diagnostic measures for depression were not used and there were no true controls [74]. A number of other studies have also documented lower depression levels following EX in a variety of chronic illnesses including chronic obstructive pulmonary disease (COPD), stroke, arthritis, and fibromyalgia [75-78]. The role of EX in reducing depression in HF has not been systematically examined in adequate samples, and few EX trials have included depression outcomes. The HFACTION trial, the largest study of the efficacy of EX in HF patients, did not include depression as a major outcome; however, the results showed that depressive symptoms may negatively influence perceived disease severity [38,79]. Currently, one randomized controlled trial (RCT) (The Understanding Prognostic Benefits of Exercise and Antidepressant Therapy for Persons With Depression and Heart Disease Study) [80] is comparing the effects of sertraline, EX, and placebo on depression and biomarkers of cardiovascular risk in patients with stable coronary artery disease (CAD) and elevated depressive symptoms.

The reason for depression reduction in the UC group is unclear but is consistent with previous research $[60,61]$. Perhaps because a number of participants lived alone, were older, and lived in rural areas, the process of data collection in itself may have positively influenced mood and lowered HAM-D scores. Also, participants may have expected that being involved in a study of depression would improve their depression severity. However, the major reason why we think patients improved in UC was that $40 \%$ had minor depression, suggesting they probably would have improved without any intervention.

\section{Limitations}

A major limitation of this study was the small size of each group, which may have prevented detection of statistically significant differences between groups. Also, as noted above, the fact that $40 \%$ of our patients had only minor depression may have reduced intervention results so that they did not significantly differ from UC. In future studies, including patients with higher HAM-D scores or addressing overlapping symptoms of depression and HF may avoid confounding depression severity with intervention efficacy. We used self-report measures for documenting physical activity; objective measures such as pedometers or accelerometers may be more beneficial in future studies for determining objective amounts of physical activity among depressed patients.

\section{Conclusions}

We found that a combined approach using CBT and EX was superior to either therapy alone for reducing depression, increasing physical function, and improving HRQOL in patients with NYHA Class II and III HF. Cognitive behavioral therapy is relatively easy to learn and can be applied in most clinical settings and targets changing negative automatic thoughts, improving coping skills, and increasing physical activity level through behavioral activation techniques.

As a component of behavioral activation, we found that use of activity logs were well received by the study participants and can also be incorporated into most practice settings with little additional expense to monitor inertia and activity levels. The EX logs were also easy to complete for participants and helped them self-monitor their EX progress, which may have contributed to better adherence rates. Use of EX is also promising, especially among less depressed patients, but may need to be augmented with incentives for EX and at a dose sufficient to increase physical function.

Finally, the study clearly documented the feasibility of a home-based CBT and EX intervention in a frail, depressed HF population. We also found that telephonic CBT and EX sessions used in the 12-week follow-up period were feasible and acceptable for most patients and may be a more cost-effective approach in future studies. Patients with HF who are experiencing moderate-to-major depression may be at high risk for further physical function decline and adverse outcomes, including higher morbidity and mortality. The findings from this study support the need for an intervention that addresses both the physical and psychological burden of depression in this vulnerable patient population.

\section{Acknowledgments}

Funding for the study was provided by the Southeast Affiliate of the American Heart Association Beginning 
Grant-in-Aid, Atlanta Clinical and Translational Science Institute at Emory University School of Medicine.

\section{References}

[1] Rumsfeld JS, Havranek E, Masoudi FA, Peterson ED, Jones P, Tooley $\mathrm{JF}$, et al. Depressive symptoms are the strongest predictors of shortterm declines in health status in patients with heart failure. J Am Coll Cardiol 2003;42:1811-7.

[2] Joynt KE, Whellan DJ, O'Connor CM. Why is depression bad for the failing heart? A review of the mechanistic relationship between depression and heart failure. J Cardiac Fail 2004;10:258-71.

[3] Katon W, Lin EH, Kroenke K. The association of depression and anxiety with medical symptom burden in patients with chronic medical illness. Gen Hosp Psychiatry 2007;29:147-55.

[4] Katon W. The impact of major depression on chronic medical illness. Gen Hosp Psychiatry 1996;18:215-9.

[5] Masoudi FA, Rumsfeld JS, Havranek EP, House JA, Peterson ED, Krumholz HM, et al, and for the Cardiovascular Outcomes Research Consortium (CORC). Age, functional capacity, and health-related quality of life in patients with heart failure. J Cardiac Fail 2004;10: 368-73.

[6] Turvey CL, Klein DM, Pies CJ. Depression, physical impairment, and treatment of depression in chronic heart failure. J Cardiovasc Nurs 2006;21:178-85.

[7] Spertus JA, McDonell M, Woodman CL, Fihn SD. Association between depression and worse disease-specific functional status in outpatients with coronary artery disease. Am Heart J 2000;140:105-10.

[8] Vaccarino V, Kasl S, Abramson J, Krumholz HM. Depressive symptoms and risk of functional decline and death in patients with heart failure. J Am Coll Cardiol 2001;38:199-205.

[9] Murberg TA, Bru E, Tveteras R, Aarsland T. Depressed mood and subjective symptoms as predictors of mortality in patients with congestive heart failure: a two-year follow-up study. Int J Psychiatry Med 1999;29:311-26.

[10] Junger J, Schellberg D, Muller-Tasch T, Raupp G, Zugck C, Haunstetter A, et al. Depression increasingly predicts mortality in the course of congestive heart failure. Eur J Heart Fail 2005;7:261-7.

[11] Rozzini R, Trabucchi M. Depression and negative outcomes in patients with heart failure. Arch Intern Med 2003;163:498.

[12] Jiang W, Kuchibhatla M, Clary GL, Cuffe MS, Christopher EJ, Alexander JD, et al. Relationship between depressive symptoms and long-term mortality in patients with heart failure. Am Heart J 2007; 154:102-8.

[13] Rutledge T, Reis VA, Linke SE, Greenberg BH, Mills PJ. Depression in heart failure a meta-analytic review of prevalence, intervention effects, and associations with clinical outcomes. J Am Coll Cardiol 2006; $48: 1527-37$.

[14] Glassman AH. Depression and cardiovascular comorbidity. Dialogues Clin Neurosci 2007;9:9-17.

[15] Wulsin LR, Evans JC, Vasan RS, Murabito JM, Kelly-Hayes M, Benjamin EJ. Depressive symptoms, coronary heart disease, and overall mortality in the Framingham Heart Study. Psychosom Med 2005;67:697-702.

[16] Morgan AL, Masoudi FA, Havranek EP, Jones PG, Peterson PN, Krumholz HM, et al, and for the Cardiovascular Outcomes Research Consortium (CORC). Difficulty taking medications, depression, and health status in heart failure patients. J Cardiac Fail 2006;12:54-60.

[17] Carney RM, Freedland KE. Depression in patients with coronary heart disease. Am J Med 2008;121(11 Suppl 2):S20-7.

[18] Carney RM, Freedland KE, Miller GE, Jaffe. Depression as a risk factor for cardiac mortality and morbidity: a review of potential mechanisms. J Psychosom Res 2002;53:897-902.

[19] Liao L, Anstrom KJ, Gottdiener JS, Pappas PA, Whellan DJ, Kitzman $\mathrm{DW}$, et al. Long-term costs and resource use in elderly participants with congestive heart failure in the Cardiovascular Health Study. Am Heart J 2007;153:245-52.

[20] Liao L, Jollis JG, Anstrom KJ, Whellan DJ, Kitzman DW, Aurigemma GP, et al. Costs for heart failure with normal vs reduced ejection fraction. Arch Intern Med 2006;166:112-8.

[21] Sullivan M, Simon G, Spertus J, Russo J. Depression-related costs in heart failure care. Arch Intern Med 2002;162:1860-6.

[22] Carels RA. The association between disease severity, functional status, depression and daily quality of life in congestive heart failure patients. Qual Life Res 2004;13:63-72.

[23] Juenger J, Schellberg D, Kraemer S, Haunstetter A, Zugck C, Herzog $\mathrm{W}$, et al. Health related quality of life in patients with congestive heart failure: comparison with other chronic diseases and relation to functional variables. Heart 2002;87:235-41.

[24] Lee DT, Yu DS, Woo J, Thompson DR. Health-related quality of life in patients with congestive heart failure. Eur J Heart Fail 2005;7: 419-22.

[25] Calvert MJ, Freemantle N, Cleland JG. The impact of chronic heart failure on health-related quality of life data acquired in the baseline phase of the CARE-HF study. Eur J Heart Fail 2005;7:243-51.

[26] Sherwood A, Blumenthal JA, Trivedi R, Johnson KS, O'Connor CM, Adams KF, et al. Relationship of depression to death or hospitalization in patients with heart failure. Arch Intern Med 2007;167:367-73.

[27] Koenig HG. Depression outcome in patients with congestive heart failure. Arch Intern Med 2006;166:991-6.

[28] Druss BG, Rask K, Katon WJ. Major depression, depression treatment and quality of primary medical care. Gen Hosp Psychiatry 2008;30: $20-5$.

[29] Fenton WS, Stover ES. Mood disorders: cardiovascular and diabetes comorbidity. Curr Opin Psychiatry 2006;19:421-7.

[30] Coletta AP, Clark AL, Cleland JG. Clinical trials update from the Heart Failure Society of America and the American Heart Association meetings in 2008: SADHART-CHF, COMPARE, MOMENTUM, thyroid hormone analogue study, HF-ACTION, I-PRESERVE, $\{$ beta $\}$-interferon study, BACH, and ATHENA. Eur J Heart Fail 2009;11:214-9.

[31] Lett HS, Davidson J, Blumenthal JA. Nonpharmacologic treatments for depression in patients with coronary heart disease. Psychosom Med 2005;67(Suppl 1):S58-S62.

[32] Iosifescu DV. Treating depression in the medically ill. Psychiatr Clin North Am 2007;30:77-90.

[33] Turvey CL, Klein DM, Pies CJ, Arndt S. Attitudes about impairment and depression in elders suffering from chronic heart failure. Int $\mathrm{J}$ Psychiatry Med 2003;33:117-32.

[34] Dekker RL. Cognitive behavioral therapy for depression in patients with heart failure: a critical review. Nurs Clin North Am 2008;43: $155-70$.

[35] Beck AT, Rush AJ, Shaw BF, Emery G. Cognitive therapy of depression. New York: Guilford Press, 1979.

[36] Beck J. Cognitive therapy: basics and beyond. New York: Guilford Press, 1995.

[37] Feldman G. Cognitive and behavioral therapies for depression: overview, new directions, and practical recommendations for dissemination. Psychiatr Clin of North Am 2007;30:39-50.

[38] Whellan DJ, O'Connor CM, Lee KL, Keteyian SJ, Cooper LS, Ellis SJ, et al. Heart failure and a controlled trial investigating outcomes of exercise training (HF-ACTION): design and rationale. Am Heart J 2007;53:201-11.

[39] Pina IL, Apstein CS, Balady GJ, Belardinelli R, Chaitman BR, Duscha BD, et al, and American Heart Association Committee on exercise, rehabilitation and prevention. Exercise and heart failure: a statement from the American Heart Association Committee on exercise, rehabilitation and prevention. Circulation 2003;107: $1210-25$

[40] Sullivan MJ, Higginbotham MB, Cobb FR. Exercise training in patients with severe left ventricular dysfunction: hemodynamic and metabolic effects. Circulation 1988;78:506-15. 
[41] Belardinelli R, Georgiou D, Scocco VBarstow TJ, Purcaro A. Low intensity exercise training in patients with chronic heart failure. $\mathrm{J}$ Am Coll Cardiol 1995;26:975-82.

[42] Itoh K, Osada N, Inoue K, Samejima H, Seki A, Omiya K, et al. Relationship between exercise intolerance and levels of neurohormonal factors and proinflammatory cytokines in patients with stable chronic heart failure. Int Heart J 2005;46:1049-59.

[43] Adamopoulos S, Coats AJ, Brunotte F, Arnolda L, Meyer T, Thompson $\mathrm{CH}$, et al. Physical training improves skeletal muscle metabolism in patients with chronic heart failure. J Am Coll Cardiol 1993;21:1101-6.

[44] Hornig B, Maier V, Drexler H. Physical training improves endothelial functions in patients with chronic heart failure. Circulation 1996;93: $210-4$.

[45] Hambrecht R, Gielen S, Linke A, Fiehn E, Yu J, Walther C, et al. Effects of exercise training on left ventricular function and peripheral resistance in patients with chronic heart failure: a randomized trial. JAMA 2000;283:3095-101

[46] Oka RK, De Marco T, Haskell WL, Botvinick E, Dae MW, Bolen K, et al. Impact of a home-based walking and resistance training program on quality of life in patients with heart failure. Am J Cardiol 2000;85: 365-9.

[47] American Psychiatric Association. Diagnostic and statistical manual of mental disorders (DSM-IV-TR). 4th ed. Washington, DC: American Psychiatric Association, 2000.

[48] Beck AT, Steer RA, Ball R, Ranieri W. Comparison of Beck Depression Inventories-IA and -II in psychiatric outpatients. J Pers Assess 1996;67:588-97.

[49] American College of Cardiology/American Heart Association task force on Practice Guidelines. Committee on evaluation and management of heart failure. Guidelines for the evaluation and management of heart failure. Circulation 1995;92:2764-84.

[50] Hamilton M. A rating scale for depression. J Neurol Neurosurg Psych 1960;23:56-62.

[51] Sheehan DV, Lecrubier Y, Sheehan K, Janavs J, Weiller E, Keskiner A, et al. The validity of the Mini International Neuropsychiatric Interview (MINI) according to the SCID-P and its reliability. European Psychiatr 1997;12:232-41.

[52] Sheehan DV, Lecrubier Y, Sheehan K, Amorim P, Janavs J, Weiller E, et al. The Mini-International Neuropsychiatric Interview (M.I.N.I): the development and validation of a structured diagnostic psychiatric interview for DSM-IV and ICD-10. J Clin Psychiatr 1998;59(Suppl 20):22-33.

[53] Guyatt GH, Sullivan MJ, Thompson PJ, Fallen EL, Pugsley R, Taylor DW, et al. The 6-minute walk: a new measure of exercise capacity in patients with chronic heart failure. Can Med Assoc J 1985;132:919-23.

[54] Demers C, McKelvie RS, Negassa A, Yusuf S. Reliability, validity, and responsiveness of the six-minute walk test in patients with heart failure. Am Heart J 2001;142:698-703.

[55] Peeters P, Mets T. The 6-minute walk as an appropriate exercise test in elderly patients with chronic heart failure. J Gerontol A Biol Sci Med Sci 1996;51:M147-M151.

[56] Hamilton DM, Haennel RG. Validity and reliability of the 6-minute walk test in a cardiac rehabilitation population. J Cardiopulm Rehab 2000;20:156-64.

[57] McDermott MM, Greenland P, Guralnik JM, Liu K, Criqui MH, Pearce WH. Depressive symptoms and lower extremity functioning in men and women with peripheral arterial disease. J Gen Intern Med 2003; 18:461-7.

[58] Rector TS, Kubo SH, Cohn JN. Patients' self-assessment of their congestive heart failure. Heart Fail 1987:198-209.

[59] Middel B, Bouma J, deJongste M, van Sonderen E, Niemeijer MG, Crijns $\mathrm{H}$, et al. Psychometric properties of the Living with Heart Failure Questionnaire (MLHF-Q). Clin Rehab 2001;15:489-500.
[60] Young J, Beck AT. Cognitive therapy scale rating manual. Philadelphia: University of Pennsylvania Center for Psychotherapy Research, 1980.

[61] Borg G. Psychophysical bases of perceived exertion. Med Sci Sports Exerc 1982;14:377-81.

[62] Polar USA. Polar heart rate monitors [Web Page].

[63] Karvonen M, Kentala K Mustalo O. The effects of training on heart rate: a longitudinal study. Ann Med Exp Biol Fenn 1957;35:307.

[64] Thase ME. Augmentation strategies for depression: history and concepts. CNS Spectr 2007;12(12 Suppl 22):3-5.

[65] American Psychiatric Association. Practice guidelines for the treatment of patients with major depressive disorder (revision). Am J Psychiatr 2000;157(Suppl 4):1-45.

[66] Glassman AH, Bigger JT, Gaffney M, Shapiro PA, Swenson JR. Onset of major depression associated with acute coronary syndromes: relationship of onset, major depressive disorder history, and episode severity to sertraline benefit. Arch Gen Psychiatr 2006;63: 283-8.

[67] Hollon SD, Jarrett RB, Nierenberg AA, Thase ME, Trivedi M, Rush AJ. Psychotherapy and medication in the treatment of adult and geriatric depression: which monotherapy or combined treatment? J Clin Psychiatr 2005;66:455-68.

[68] Kostis JB, Rosen RC, Cosgrove NM, Shindler DM, Wilson AC. Nonpharmacologic therapy improves functional and emotional status in congestive heart failure. Chest 1994;106:996-1001.

[69] Barbour KA, Edenfield TM, Blumenthal JA. Exercise as a treatment for depression and other psychiatric disorders: a review. J Cardiopulm Rehabil Prev 2007;27:359-67.

[70] Brosse AL, Sheets ES, Lett HS, Blumenthal JA. Exercise and the treatment of clinical depression in adults: recent findings and future directions. Sports Med 2002;32:741-60.

[71] Dunlop BW, Self RL. Exercise for depression: efficacy, safety and clinical trial implications. Psychopharmacol Bull 2008;41: $65-75$.

[72] Trivedi MH, Greer TL, Grannemann BD, Chambliss HO, Jordan AN. Exercise as an augmentation strategy for treatment of major depression. J Psychiatr Pract 2006;12:205-13.

[73] Blumenthal JA, Babyak MA, Doraiswamy PM, Watkins L, Hoffman $\mathrm{BM}$, Barbour KA, et al. Exercise and pharmacotherapy in the treatment of major depressive disorder. Psychosom Med 2007;69: $587-96$

[74] Milani RV, Lavie CJ. Impact of cardiac rehabilitation on depression and its associated mortality. Am J Med 2007;120:799-806.

[75] Goldenberg DL. Multidisciplinary modalities in the treatment of fibromyalgia. J Clin Psychiatry 2008;69(Suppl 2):30-4.

[76] Kozora E, Emery C, Kaplan RM, Wamboldt FS, Zhang L, Make BJ. Cognitive and psychological issues in emphysema. Proc Am Thorac Soc 2008;5:556-60.

[77] Karmisholt K, Gotzsche PC. Physical activity for secondary prevention of disease. Systematic reviews of randomised clinical trials. Dan Med Bull 2005;52:90-4.

[78] Lai SM, Studenski S, Richards L, Perera S, Reker D, Rigler S, et al. Therapeutic exercise and depressive symptoms after stroke. J Am Geriatr Soc 2006;54:240-7.

[79] Gottlieb SS, Kop WJ, Ellis SJ, Binkley P, Howlett J, O'Connor C, et al, HF-ACTION Investigators. Relation of depression to severity of illness in heart failure (from Heart Failure And a Controlled Trial Investigating Outcomes of Exercise Training [HF-ACTION]). Am J Cardiol 2009;103:1285-9.

[80] Blumenthal JA, Sherwood A, Rogers SD, Babyak MA, Doraiswamy PM, Watkins L. Understanding prognostic benefits of exercise and antidepressant therapy for persons with depression and heart disease: the UPBEAT study - rationale, design, and methodological issues. Clin Trials 2007:4:548-59. 